\title{
COLABORACIÓN MÉXICO-ESTADOS UNIDOS EN MEMS/NEMS
}

\author{
Guillermo Foladori*; Edgar Záyago Lau** \\ Remberto Sandoval ${ }^{* * *}$; Richard Appelbaum*** \\ Rachel Parker*****
}

RESUMEN: Este artículo tiene el objetivo de explorar la cooperación binacional entre México y Estados Unidos sobre Sistemas Micro-Electro Mecánicos (MEMs) y Sistemas Nano-Electro Mecánicos (NEMs). Es una cooperación que está en sus albores, pues tiene menos de una década. Sin embargo, su desarrollo muestra al menos dos cuestiones que merecen destacarse. Una de ellas es la coincidencia de intereses militares y civiles en la investigación y desarrollo $(\mathrm{I}+\mathrm{D})$ de esta tecnología. Este propósito común hace posible la formación de recursos humanos y la especialización de civiles mexicanos dentro de instituciones estadounidenses con orientación militar; pero levanta la duda sobre el grado de independencia de la agenda de investigación de mems en México. Otra cuestión a tener en cuenta es que las instituciones mexicanas son capaces de desarrollar nuevas áreas tecnológicas con poca tradición, siempre y cuando la voluntad política esté presente.

palabras Claveः nems, mems, nanotecnología, México, Estados Unidos.

ABSTRACT: This article seeks to explore bi-national collaboration between Mexico and the United States in relation to mems/Nems. This cooperation is in its initial stages; it has existed for less than a decade. However, its development shows at least two aspects that merit consideration. One has to do with the coincidence of military and civil interests in the research and development $(\mathrm{R}+\mathrm{D})$ of this technology. This common purpose makes possible the formation of human resources and the specialization of Mexicans in US institutions with a military orientation; but it raises doubt with regard to the degree of independence of the research agenda on MEMs in Mexico. Another aspect that needs to be considered is that Mexican institutions are only capable of developing new technological areas if there is political will to do so.

KEY WORDS NEMS, MEMS, Nanotechnology, Mexico, US.

* Docente investigador de la Unidad Académica de Estudios del Desarrollo de la Universidad Autónoma de Zacatecas, México.

** Docente investigador de la Unidad Académica de Estudios del Desarrollo de la Universidad Autónoma de Zacatecas, México.

*** Docente investigador de la Unidad Académica de Ingeniería Eléctrica de la Universidad Autónoma de Zacatecas, México.

**** Docente investigador de la University of California at Santa Bárbara, California, Estados Unidos.

***** Investigadora de Science and Technology Policy Institute, Washington, Estados Unidos.

Nota: Esta investigación fue financiada por la beca uc Mexus-Conacyt CN 10-420. 


\section{INTRODUCCIÓN}

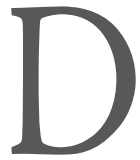

esde que en 2000 se puso en marcha la Iniciativa Nacional de Nanotecnología de Estados Unidos, los Sistemas Micro-Electro Mecánicos (MEMs, por sus siglas en inglés) se han asociado con la nanotecnología. Por su tamaño, los mems son micro, no nano, y no implican diferentes atributos físicos o químicos como las aplicaciones nano; ${ }^{1}$ sin embargo, hay tres elementos que vinculan a estos sistemas con las nanotecnologías. En primer lugar, los MEMS se entremezclan con la tendencia hacia la miniaturización ya presente en la combinación de éstos con nanoestructuras. El acrónimo Nems (Sistemas Nano-Electro Mecánicos, por sus siglas en inglés) es utilizado para describir dispositivos que combinan funciones mecánicas y eléctricas en nanoescala, y ejemplifican la transición de MEMs en NEMS. En segundo lugar, debemos prestar atención a la infraestructura y equipamiento. Los procesos de fabricación de mems incluyen el uso de cuartos limpios y microscopios de fuerza atómica (ATM), y los NEMS también requieren una infraestructura y equipo similar.

El tercer elemento es la materia prima utilizada. La principal materia prima que se utiliza en la fabricación de mems es el silicio, pero hay muchos otros materiales procedentes de procesos nano-habilitados, a partir de los cuales se espera obtener ventajas. Sabemos que la materia a escala nanométrica tiene diferentes características químicas, físicas y mecánicas que los mismos elementos en escala mayor; por tanto, una vez que estas ventajas se hacen evidentes, se logra la transición de mEMS a NEMS. En cuanto al financiamiento público, la Iniciativa Nacional de Nanotecnología de Estados Unidos gasta enormes fondos en MEMs y sensores, así

${ }^{1}$ La tecnología mems implica trabajar en la microescala (1 a 100 micrómetros); un micrómetro es la millonésima parte de un metro. La nanotecnología implica trabajar a una escala menor a 100 nanómetros, donde moléculas y átomos individuales pueden diseñarse para tener nuevas propiedades (un nanómetro es la milmillonésima parte de un metro; por dar una referencia, una hoja de periódico tiene aproximadamente 100,000 nanómetros de espesor). Hay 1,000 nanómetros en un micrómetro. Para mayor información véase la página web de la Iniciativa Nacional de Nanotecnología de Estados Unidos: http://www.nano.gov/nanotech-101/what/definition. 
como en laboratorios con equipos utilizados por y para éstos (Materials Modification Inc., s/f; Plunkett, 2010), de manera que también desde el punto de vista financiero, MEMS y NEMS son considerados conjuntamente.

Este artículo tiene el objetivo de explorar la cooperación binacional entre México y Estados Unidos acerca de MEms y NEMS. Es una cooperación que está en sus albores, tiene menos de una década; sin embargo, su desarrollo muestra al menos dos cuestiones que merecen destacarse. Una de ellas es la coincidencia de intereses militares y civiles en la investigación y desarrollo (I+D) de esta tecnología. Este propósito común hace posible la formación de recursos humanos y la especialización de civiles mexicanos dentro de instituciones estadounidenses con orientación militar; pero levanta la duda sobre el grado de independencia de la agenda de investigación de mems en México. Otra cuestión a tener en cuenta es que las instituciones mexicanas son capaces de desarrollar nuevas áreas tecnológicas con poca tradición, siempre y cuando la voluntad política esté presente. Empero, en México la tecnología mems no ha ido más allá de los primeros pasos, y llegar a un nivel de proceso de producción es todavía incierto.

La investigación adoptó un enfoque de sociología política para analizar la transferencia de tecnología entre Estados Unidos y México. Para ello se combinaron tres tipos de información. En primer lugar se realizó un análisis de las características materiales de la tecnología estudiada (MEMS / NEMS) con el propósito de identificar los requisitos materiales que debe cumplir dicha tecnología para establecerse en el país receptor en un contexto sin antecedentes científico-técnicos. En segundo lugar se analizó la trayectoria política que ha seguido la tecnología en Estados Unidos, el país que la transfiere a México. Esto resultó importante debido al carácter de doble uso (civil y militar) que esta tecnología adquirió en Estados Unidos. En tercer lugar se analizó la institución que ejerció como promotor de transferencia tecnológica, la Fundación MéxicoEstados Unidos para la Ciencia (Fumec). Este análisis llevó a resaltar la influencia del contexto político de ambos países a comienzos del siglo, 
y el cambio que sufrió dicha institución como resultado. Por último se expuso el resultado de la propuesta de incentivar la investigación y desarrollo de mems y nems en México.

La información del artículo es resultado de la sistematización de información técnica, histórica y documental. Se basa en artículos tecnocientíficos sobre tecnología; en la revisión de documentos oficiales, como los informes anuales de actividad de la agencia promotora de la tecnología en México; en información hemerográfica y en la revisión de las páginas Web de las instituciones involucradas.

Este artículo se organiza de la siguiente manera: la primera parte explica los aspectos generales de los MEMS, NEMs y las fases de $\mathrm{I}+\mathrm{D}$ requeridas para la fabricación de prototipos de los mismos. Éste es un tema clave para entender los requisitos y el equipo necesario para la fabricación de estos sistemas. En la segunda sección se expone la finalidad de doble uso (civil y militar) de la tecnología mems. Por un lado, son utilizados por el sector civil, por el otro son de interés central para las instituciones militares. Esta doble característica ha permitido la creación de programas conjuntos entre los laboratorios militares y grupos civiles de investigación en Estados Unidos y México. La tercera sección analiza el alcance y acciones institucionales de la Fumec, para la cual los Nems son una de las áreas más importantes de la colaboración científica. En esta sección se muestra el entorno sociopolítico en el que se fomentaron los acuerdos, así como los cambios por los que tuvo que pasar la Fumec para apoyar la innovación industrial. En la cuarta sección se describe cómo dicha fundación organizó la transferencia de tecnología relacionada con MEMS a las instituciones mexicanas en colaboración con organismos con sede en Estados Unidos. También trazamos un mapa de las capacidades actuales, relacionadas con los mems y cómo se distribuyen en México. Concluimos con un resumen de los hallazgos y resaltamos los importantes avances en ciencia y tecnología (сут) que se pueden lograr cuando existe compromiso político. 


\section{CARACTERÍSTICAS GENERALES DE LOS MEMS/NEMS}

$$
\text { ¿Qué son los MEMS/NEMS? }
$$

Los sistemas micro-electromecánicos o nano-electromecánicos (MEMS/ NEMS) son pequeños dispositivos conformados por componentes eléctricos, electrónicos y mecánicos de tamaño micro o nanoscópico que pueden ir de unos cuantos décimos de micrómetros hasta varios milímetros, dependiendo de la complejidad del sistema. Éstos son capaces de interactuar con procesos físicos, químicos, biológicos, etcétera, en el mundo microscópico de tal manera que puedan detectar o manipular ciertos parámetros físicos en micro-escala; incluso pueden generar efectos detectables en escala mayor (Anónimo, 2007; Prime Faraday, 2002). El término mems fue acuñado en Estados Unidos, mientras que en Europa son conocidos como micro-sistemas (Microsystems Technology, MST) y en Japón como dispositivos micro-maquinados. El término mems es el más difundido, por lo que es empleado mundialmente para identificar a estos dispositivos (Banks, 2006; Maluf, 2000; Prime Faraday, 2002; Senturia, 2000). Por NEMs se entiende aquellos dispositivos de tamaño por debajo de los 100 nanómetros que pueden explotar las ventajas del tamaño y de las propiedades físico-químicas que los materiales expresan en tal dimensión. ${ }^{2}$

Para poder dar una idea más clara de lo que son, tomemos en cuenta su contraparte macroscópica: los sistemas electromecánicos. Consideremos un sistema compuesto por una cisterna, un tinaco, una bomba y un electro-nivel. El sistema en conjunto funciona de tal manera que nunca falte agua en el tinaco. Para lograrlo, el electro-nivel ubicado en el tinaco detecta la cantidad de agua; al ser menor a cierto nivel, enciende la bomba ubicada en la cisterna, la cual provee de agua al tinaco. El nivel

\footnotetext{
2 «(...) cuando las partículas son creadas con dimensiones entre 1-100 nanómetros (donde sólo pueden ser 'vistas' con poderosos microscopios especializados), las propiedades de los materiales cambian significativamente respecto a estos mismos en escala macro. Esta es la escala donde los llamados efectos cuánticos gobiernan el comportamiento y las propiedades de las partículas (...) al cambiar el tamaño de la partícula, un científico puede, literalmente, ajustar una propiedad material acorde a su interés» (nano,gov, s/f).
} 
del agua comenzará a subir hasta que el electro-nivel detecte que es suficiente (antes de desbordar el tinaco y derramar el agua) y apagará la bomba. En este sistema podemos identificar tres partes importantes: el detector (electro-nivel), el actuador o manipulador (la bomba) y el control (las conexiones que prenden y apagan la bomba). De la misma manera, un MEMs puede contener alguno o los tres componentes en escala microscópica e interactuar con un sistema en escala macroscópica. En un sentido más amplio, estos sistemas consisten en microestructuras (o arreglos de microestructuras), micro-detectores, micro-actuadores y microelectrónica, todo en un solo circuito integrado de silicio (Prime Faraday Partnership, 2002). Algunos ejemplos de MEMs son los acelerómetros ubicados en las bolsas de aire de los autos o en algunos Smartphones, sensores de presión en las llantas de los autos, los populares inyectores de tinta en las impresoras, etcétera.

Los MEMS se fabrican empleando la tecnología de los circuitos integrados de silicio, con la diferencia de que los componentes micro-mecánicos son construidos empleando manipulaciones sofisticadas de este elemento $\mathrm{u}$ otros materiales, usando procesos de micro o nano-fabricación, como el micro-maquinado de superficie, micro-maquinado de volumen, entre otros. Mientras la tecnología de los circuitos integrados explota las características eléctricas, los MEMs explotan las características mecánicas y eléctricas del silicio y de otros materiales (Prime Faraday, 2002).

Frente a los esquemas macroscópicos, las ventajas de los MEMs son inherentes al tamaño: peso reducido, consumo de baja potencia, portabilidad y alta funcionalidad; resultan apropiados para grandes volúmenes de producción a bajo costo y aportan confiabilidad, nuevas soluciones y nuevas aplicaciones. Además de esto, cabe resaltar la posibilidad de integrar mayor «inteligencia» en un solo dispositivo, lo que incrementa la confiabilidad ya que se tienen menos partes y por lo tanto menos posibles fallas en el sistema. Además, la miniaturización hace posible la explotación de nuevas aplicaciones y nuevos mercados, como los controles de los videojuegos modernos como el Wii de Nintendo, las aplicaciones 
de los acelerómetros en los smartphones, la identificación de ADN en biotecnología o los filtros mecánicos para comunicaciones inalámbricas (MNS, s/f).

\section{El proceso de producción de los MEMS}

El desarrollo y producción de MEMs de bajo costo es un proceso que requiere una inversión importante de recursos económicos, temporales y humanos, por lo que sólo se justifica para mercados cuya demanda sea grande (del orden de los 10 millones de dispositivos). Pocos productos basados en esta tecnología pueden pasar de volúmenes de prototipos a producción a gran escala (Da Silva, Giasolli, Cunningham y DeRoo, 2002). Algunos ejemplos son los giroscopios, sensores de presión, inyectores de tinta, acelerómetros, redes de conmutación en $\mathrm{RF}$ (radio frecuencia) u ópticas, almacenamiento de datos y sistemas de análisis químicos desechables (Fedder, 1999; Mukherjee, 2003). El estudio del ciclo de un producto que contiene MEMs es importante para poder entender la cadena de valor de los mismos, ya que la última va inmersa en la primera. Un flujo típico consiste en las siguientes cinco grandes etapas:

Diseño y modelado Micro-manufactura $\rightarrow$ Caracterización $\rightarrow$ Confiabilidad $\rightarrow$ Prototipo

Consideremos el desarrollo de un smartphone con un giroscopio mems. La primera etapa la denominamos diseño y modelado; se trata de un periodo totalmente virtual, que sólo requiere del equipo de cómputo necesario y el conocimiento de los requisitos del producto. Esta característica permite que exista una gran cantidad de centros de investigación que no requieren de mayor volumen de capital invertido, a diferencia de las etapas subsiguientes que incorporan procesos materiales que, por el tamaño micro y nano de los dispositivos, obliga a cuartos limpios y equipo sofisticado. Al tratarse de una etapa virtual, el diseño y modelado 
es fácilmente subcontratado a pequeñas empresas por las grandes corporaciones.

El primer paso dentro de esta etapa es definir las especificaciones del producto; es decir, las características que debe de tener para lograr un impacto positivo. En el caso del smartphone: que tenga una pantalla táctil de ciertas dimensiones, ligero o con un peso menor a cierto límite, con capacidad de reaccionar a cambios de posición, etcétera. En seguida se hace el diseño del producto basado en estas especificaciones; en esta parte se divide el proceso de desarrollo del producto en partes más pequeñas y manejables donde se definen los componentes del producto: pantalla, equipo de comunicación, equipo de control, giroscopio, micrófono, bocina y demás. Aquí es donde se ve la necesidad de emplear un mems para lograr una innovación, ya sea en tamaño, consumo de potencia o aplicación, a fin de hacer más atractivo el producto; para el smartphone analizado, la utilización del giroscopio responde a cambios en la orientación del dispositivo. Del componente mems (giroscopio) se desprenden las especificaciones de la tecnología que debe emplearse; es decir, su principio de operación. En este caso, pudiera estar basado en un funcionamiento capacitivo con partes móviles. Hasta este punto, el desarrollo del producto puede ser llevado por una compañía subcontratada.

El segundo paso en la etapa de diseño y modelado implica determinar las especificaciones y trazar el plano de disposición de los elementos del MEMs (microestructuras e.g. bisagras, peines, engranes). Es importante resaltar que las medidas del plano son en micras, y que se tiene un plano por cada capa de material que se utilice en el proceso de manufactura. Si se emplea el proceso summit v de los Laboratorios Nacionales Sandia, el dispositivo puede estar compuesto hasta por cinco planos o capas.

Una vez finalizada la etapa de diseño y modelado, se pasa a la segunda etapa de micro o nano manufactura. Esta etapa incluye la manipulación material. El proceso de desarrollo deja de ser totalmente virtual y se 
requiere de equipo sofisticado, cuartos limpios y medidas de control y seguridad.

Se puede iniciar un proceso de micro-manufactura según el procedimiento técnico seleccionado (por ejemplo, Liga, summit v, micromecanizado de volumen). Se entiende que a la salida de la etapa del proceso de manufactura se tiene un мемs empaquetado y con conexiones al exterior, con lo cual se puede introducir el dispositivo (giroscopio) en la arquitectura del producto final. En el caso del smartphone se tienen las conexiones del giroscopio con un micro-controlador que cambia la orientación del desplegado de la pantalla.

La tercera etapa en el desarrollo de este producto es la caracterización, donde se obtiene la respuesta del mems en funcionamiento a señales de excitación, de tal manera que se puede comprobar si responde de acuerdo con los parámetros de diseño establecidos; es decir, se valida el modelo, se calibran los dispositivos para el funcionamiento adecuado, se hace el análisis de respuesta en presencia de ruido, entre otros. En el caso del giroscopio, se pueden hacer pruebas de sensibilidad a diferentes magnitudes o de cambios de orientación para así, medir la intensidad de su respuesta (puede ser en forma de una amplitud de voltaje).

La cuarta etapa del desarrollo de productos mems es la prueba de confiabilidad. Se somete el dispositivo a condiciones de funcionamiento extremas para localizar las posibles fallas que se puedan presentar con el uso, tales como fallas estructurales, estrés en los materiales, desgaste, temperaturas extremas, entre otras. Para el smartphone, esta etapa puede ir desde respuesta a señales de RF, hasta la calidad en la señal de audio, pasando por la sensibilidad a los cambios de orientación de la pantalla producidos por la señal generada por el giroscopio. Asimismo, se realizan pruebas de confiabilidad en el producto, donde se estudian las posibles fallas y de esta manera poder corregir el diseño original, pudiendo cambiar las especificaciones originales del producto y reiniciar el ciclo de desarrollo.

Una vez que se ha finalizado con la prueba de confiabilidad puede ser creado un prototipo, que es la etapa final. El prototipo es un MEMS que puede ser producido a gran escala por el fabricante final. Un tercio de 
la producción mundial de estos sitemas se concentra en cuatro grandes firmas: Texas Instruments (10\%), Hewlett-Packard (9.4\%), Robert Bosch (7.4\%) y STMicroelectronics (7.0\%) (Yolé, 2011).

Las etapas no son necesariamente lineales, sino que en cada una se puede volver a las anteriores. Esto se puede ver como un proceso de prueba y error. Así, varios ciclos involucran al proceso de manufactura, provocando que el tiempo de producción se alargue, principalmente cuando se está en la etapa de prueba de los prototipos (Da Silva et al., 2002; Fedder, 1999). Entre mayor cantidad de datos se recopilen en las etapas de caracterización y pruebas de confiabilidad, mejor se podrá corregir el diseño, validar el modelo empleado y evitar repetir de manera innecesaria la fabricación, con la finalidad de reducir el tiempo y los costos de desarrollo del dispositivo (Gandhi, s/f; Lawton, 1999). Algunas herramientas especializadas ayudan a mejorar esta etapa simulando el proceso de fabricación (por ejemplo, IntelliSense o Coventor Suite). ${ }^{3}$

Estas etapas representan el proceso de investigación y desarrollo hasta llegar al prototipo. Cuando todo el proceso está probado, entonces se aplica industrialmente y el regreso a etapas anteriores no sucede. Este tipo de proceso lo podemos ver en productos populares como los desarrollados por la compañía Apple, tales como el iPod, iPhone (un smartphone) y el iPad, que presentan versiones nuevas cada año y así el ciclo se repite para mejorar el diseño y ventajas de los dispositivos; aunque también se puede ver como una manera de acortar el ciclo de rotación del capital y presionar a los usuarios para actualizar su producto.

\section{MEMS/NEMS COMO UNA TECNOLOGÍA DE DOBLE USO (CIVIL Y MILITAR)}

Los MEMS comerciales aparecieron por primera vez en ordenadores e impresoras de inyección de tinta en la década de 1980. Desde el comienzo de la

${ }^{3}$ Por ejemplo IntelliSense, que promete «Soluciones Totales en mems» (http://intellisense.com/). 
década de 1990, el gobierno de Estados Unidos invirtió importantes fondos para la investigación en los mems con fines militares. La Oficina de la Fuerza Aérea de Investigaciones Científicas (AFOSR, por sus siglas en inglés) y la Agencia de Investigación Avanzada de Proyectos de Defensa (DARPa, por sus siglas en inglés) financiaron proyectos en laboratorios militares en este campo. Los Laboratorios Nacionales Sandia (SNL, por sus siglas en inglés) fueron uno de los primeros en recibir considerables fondos públicos para la investigación en мемs, y a finales de la década de 1990 habían desarrollado procesos técnicos para producir éstos en capas ( «SUMMIT» technology). Un informe del Departamento de Defensa (DoD) calcula que en 1995 el gobierno de Estados Unidos invirtió 35 millones de dólares en I+D para MEMs, con 30 millones de éstos dirigidos a instituciones militares (Department of Defense, 1995).

Los SNL operan bajo el marco propiedad del gobierno/operado por contratistas (Government-Owned/Contractor Operated, Goco); es decir, conjuga la propiedad estatal con la administración privada ${ }^{4}$ Luego de pasar por distintas administraciones, los SNL actual son operados por Lockheed-Martin, el mayor productor mundial de armas: más de $70 \%$ de sus ingresos provienen de la venta de armas. El presupuesto anual de estos laboratorios es de aproximadamente 2.5 mil millones de dólares, de los cuales alrededor de $60 \%$ es suministrado por el Departamento de Energía (DoE) (Sandia National Laboratories, s/f). A partir de la década de los noventa, los SNL comenzaron a investigar intensamente en MEMS Y NEMS.

Su reducido tamaño otorga a los MEMS una importancia estratégica en la industria militar, especialmente en la producción de armas inteligentes de precisión. En 2001, el sitio web de Forbes señaló que el gobierno de Estados Unidos había invertido unos 200 millones de dólares anuales en éstos a través de dos organismos: la DARPA y los SNL. El director de los SNL dijo: «todo lo que es bueno para los mems es bueno para la

${ }^{4}$ El primer goco fue el Alamos National Laboratory, operado por la Universidad de California y una parte del Proyecto Manhattan, que creó la bomba atómica durante la Segunda Guerra Mundial. 
defensa nacional», mostrando la importancia estratégica militar de dichos sistemas (Freiburghouse, 2001).

El impulso que dio la industria militar a los mems ha sido un acelerador importante en la diversificación de la tecnología para uso civil. Un director vinculado a DARPA señaló: «En 1992, había poca participación de la pequeña industria y virtualmente ninguna infraestructura de fabricación de MEMs en ninguna parte del mundo. Las inversiones de DARPA en MEMS han generado esa infraestructura» (citado por Rhea, 2000).

Los mems son una tecnología de doble uso, y aunque las compras militares de la tecnología son menos que las civiles, hay dos factores de la industria militar que tienen un impacto sobre el sector civil. El primero es la eficiencia, ya que la industria militar no se rige por la tasa de ganancia sino más bien por el alto rendimiento. La segunda es la madurez, que en el sector civil implica un estancamiento o caída de los beneficios cuando se llega a tal fase, mientras que en el ámbito militar no tiene ningún efecto de estancamiento en la investigación.

A su vez, la industria civil es importante para el sector militar por tres factores. Uno es la prueba extendida a través de diversos sectores de aplicación. El director del Centro de Ciencia de Microsistemas, Tecnología y Componentes en los SNL dijo: «Antes de poder usar mems y microsistemas en sistemas críticos de armas, hay que demostrar que se pueden fabricar y son confiables. La mejor manera de demostrar esto es comercializarlos y utilizarlos en productos de uso diario» (Sandia National Laboratories, 2001a).

Otro factor es la elaboración de la infraestructura de producción a gran escala que el sector civil puede cumplir, aunque el objetivo final del sector militar sea el armamento. Esto fue destacado por el administrador del proyecto de MEMS de los SNL: «A la larga, Sandia quiere usar mems en los sistemas de armas. Pero Sandia no puede fabricar todas las piezas necesarias por sí mismo, por lo que el laboratorio está ofreciendo su tecnología MEMS y servicios propios de fabricación para la industria, con la esperanza de sembrar el mercado de mems» (Matsumoto, 1999). 
En 1998 fue creado el Sandia Science E Techcnology Park, un centro asociado con las empresas implicadas en la transferencia de tecnología. En 2001 se hizo un acuerdo con Ardesta para la producción y venta de MEMS, con la tecnología summit desarrollada en los SNL (Sandia National Laboratories, 2001b). Se estableció un programa permanente de cursos y capacitación en tecnología summit para uso comercial, conocido como samples (McBrayer, 2000), y el diálogo comenzó con la Fumec (Fundación México-Estados Unidos para la Ciencia) para iniciar el proyecto mems en México.

El tercer factor es la reducción de costos. En un artículo publicado en 2003 por la revista Military $\mathcal{E}$ Aerospace Electronics se dice que:

Los desarrollistas militares y contratistas también están buscando reducir costos ofreciendo algunas de las tecnologías evolutivas MEMs a los usuarios comerciales, tales como la industria del automóvil, esencialmente completando el círculo de desarrollo, así como alguna tecnología mems provenía de ese sector originalmente. «Garantizamos que la aplicación militar de la tecnología no ha proliferado, por supuesto, pero en la industria automotriz la exactitud que se busca no está ni cerca de lo que los militares lo requieren», dice Panhorst [gerente de programas de mems en el centro de Picatinny del Ejército] de la mems imu (Wilson, 2003).

Las sinergias entre los intereses civiles y militares acerca de la tecnología de doble uso de los mems facilitaron la colaboración México-Estados Unidos en la materia. En 1998, un oficial de alto rango del gobierno de Ronald Reagan creó y dirigió el Advanced Concept Group (ACG) al interior de los SNL con el objetivo de enfrentar los problemas del terrorismo y la seguridad interna mediante el desarrollo socioeconómico de la frontera entre México y Estados Unidos basado en parques de alta tecnología. ${ }^{5}$ Esto estaba lejos de ser una idea nueva. Desde la firma del Tratado de Libre Comercio de América del Norte (TlCAN), diversos

${ }^{5}$ Gerry Jonas era Vicepresidente y Director Científico en los Laboratorios Nacionales Sandia. 
acuerdos binacionales fueron firmados entre los estados fronterizos de México y Estados Unidos para fomentar el desarrollo económico de una manera coordinada. La especificidad de la propuesta del SNL fue apoyar la creación y la investigación en alta tecnología, algo que las maquiladoras no proporcionaban. Para lograr este fin se necesitaba una contraparte mexicana. La Fumec, institución binacional sin fines de lucro orientada al desarrollo de сут, sirvió para realizar las conexiones con el gobierno mexicano y apoyó la iniciativa para crear el Laboratorio Bi-Nacional Sustentable (BNSL).

El BNSL inició sus operaciones en 2003, a pesar de que fue lanzado oficialmente en 2005. Es una «organización bi-nacional sin fines de lucro que crea y promueve empresas de base tecnológica a lo largo de la frontera México-Estados Unidos, sean éstas de reciente creación, medianas o pequeñas, o incluso grandes empresas bien establecidas». En su inauguración, el vicepresidente de los SNL dijo: «Ésta será una oportunidad maravillosa en los esfuerzos de colaboración técnica para mejorar la seguridad en la frontera (...) Ésta es una oportunidad perfecta para dar seguimiento a trabajar con Canadá y México para fomentar un enfoque continental en materia de terrorismo» (Sandia National Laboratories, 2005). Aunque lleva el nombre de «Laboratorio», el BNSL es una agencia de comercialización de tecnología, vinculado con muchas instituciones de сут en Estados Unidos y México.

El acuerdo para la aplicación del BNSL fue impulsado del lado estadounidense por el Departamento de Comercio y la Agencia de Desarrollo Económico, el Departamento de Desarrollo Económico del Estado de Nuevo México y por los Snl, que presentaron el plan. La contraparte mexicana fue el Consejo Nacional de Ciencia y Tecnología (Conacyt), bajo la dirección del entonces presidente de México Vicente Fox. Las negociaciones fueron manejadas por Fumec (Sandia National Laboratories, 2005). En la actualidad, el BNSL trabaja en el área de mems y NEMS, combustibles limpios, nanomateriales y tecnologías ambientales. 


\section{LA FUNDACIÓN MÉXICO-ESTADOS UNIDOS PARA LA CIENCIA}

La Fumec fue creada en 1993 para promover y apoyar la colaboración de сут entre México y Estados Unidos. Fue diseñada por el equipo del congresista estadounidense George E. Brown Jr. (demócrata de California), quien dirigió el Comité de Ciencia, Tecnología y Espacio de la Cámara de Representantes de Estados Unidos.

El equipo de Brown entendió que el fin de la Guerra Fría requería una nueva relación entre Estados Unidos y el mundo en desarrollo; una relación basada en una colaboración en сут, donde los países en desarrollo podrían establecer sus propios objetivos cambiando la historia de la asistencia técnica de Estados Unidos desde la Segunda Guerra Mundial que, según dijeron, no ayudaba a promover un desarrollo independiente de capacidades en esos países (Brown y Sarewitz, 1991). Brown Jr., un pacifista, vio el final de la Guerra Fría como una oportunidad para impulsar la investigación científica más allá de los intereses militares (Brown, 1993).

Esta propuesta surgió en un contexto donde el mundo reconocía que los países que invierten sostenidamente en I+D alcanzan logros significativos, como fue el caso de Taiwán, Corea del Sur y Tailandia; pero para ello había que garantizar, de acuerdo con Brown y Sarewitz, agendas de investigación científica independientes en los países: «Lo que necesitamos son nuevos enfoques que alienten a las naciones en desarrollo para definir sus propias agendas de I+D y luego ponerlas en práctica en colaboración con el mundo desarrollado» (Brown y Sarewitz, 1991: 71).

Aplicado a América Latina, que acababa de salir de la «década perdida» de 1980, esta idea requería de creatividad para conseguir apoyo financiero. En la propuesta se sugirió copiar lo que se aplicaba en el área ambiental. Existían programas que cambiaban deuda extranjera latinoamericana a precios de mercado, con la moneda del propio país, por protección al medio ambiente. La idea era aplicar la misma política de intercambio de deuda externa, pero en este caso por сут en lugar de medio ambiente. 
México sería el caso piloto y la National Science Foundation (Fundación Nacional para la Ciencia) debía apoyar con un fondo especial: «En el Congreso, la nueva legislación (H R 3215, la Ley Interamericana de Cooperación Científica de 1991) se ha introducido para establecer una dotación binacional de ciencia entre Estados Unidos y México en 1992 y permitir a la National Science Foundation conceder becas de intercambio de deuda-para-la ciencia» (Brown y Sarewitz, 1991: 76).

Aunque la fuente de financiamiento no fue intercambio de deuda por ciencia, sino más bien un acuerdo de colaboración, Fumec fue creado en 1992 como un organismo no gubernamental con una junta directiva compuesta por 10 miembros, 5 de cada uno de los países. México optó por designar representantes que provenían de la Academia de Ciencias, Medicina, Ingeniería, el Conacyt y el coordinador de la Junta Consultiva de Ciencias de la Presidencia. Estados Unidos envió a representantes del Comité de la Cámara de Representantes del Espacio, la Ciencia y la Tecnología, el Instituto Smithsoniano, la Academia Nacional de Ciencias, el Instituto de Medicina y la Academia Nacional de Ingeniería. México y Estados Unidos compartieron a partes iguales los costes de financiación de inicio (Fumec, 1997). ${ }^{6}$

Entre 1993 y 2001, los proyectos centrados en sustentabilidad, salud pública y problemas socioeconómicos fueron prioritarios. También se invirtió en la formación de especialistas en políticas y estrategias de сут (Fumec, 1999). La contraparte de Estados Unidos fueron, principalmente, las universidades. En el Informe de Actividades 2001-2002 de la Fumec, los diferentes proyectos se agruparon de acuerdo con las áreas programáticas: salud y medio ambiente, desarrollo industrial sostenible y el desarrollo y recursos humanos en ciencia y tecnología (Fumec, 2002).

Sin embargo, desde 2001, dos años después de la muerte de Brown, comienza un importante cambio de política dentro de la Fumec. La

\footnotetext{
${ }^{6}$ Algunos de los miembros del Consejo de Gobernadores son directamente nombrados por el Presidente de cada país, lo que lleva consigo un cierto grado de estrategia política, y algunos directores de Fumec y los miembros de su Junta Directiva también eran directores de las corporaciones militares de eu y miembros del Consejo de los snl, creando alianzas personales entre las dos instituciones.
} 
innovación tecnológica pasó a ser una frase clave, y el grupo industrial con centro geográfico en la zona fronteriza cerca del Cluster Industrial Paso del Norte, donde los SNL tienen su sede, se convirtió en estratégico. Los SNL se consolidaron como el socio estadounidense de muchos de estos proyectos. El objetivo inicial de los SNL fue la puesta en marcha del Laboratorio Binacional de Sustentabilidad.

La iniciativa para desarrollar mems en México la presenta la Fumec en 2000 y 2001, durante talleres sobre esta tecnología organizados por la propia fundación, los laboratorios Sandia y la Universidad de Texas en El Paso (Fumec, 2002). En estos talleres se identificaron las capacidades científicas y tecnológicas para desarrollar mems en la zona fronteriza de El Paso y Ciudad Juárez. Los resultados obtenidos demostraron que existían pocas capacidades científicas del lado mexicano, pero que existían diversas industrias interesadas en los mems. La Fumec tuvo que enfrentar dos retos: la capacitación de recursos humanos y la construcción de capacidades tecnológicas especializadas en mems (RoblesBelmont, 2010). A partir de estos requerimientos nació el programa de microsistemas de Fumec, el cual centró sus esfuerzos en la implementación de sistemas MEMS. Posteriormente amplió su espectro para incluir sistemas embebidos y diseños en FPGA (Field Programmable Gate Array), que es una especie de hardware reconfigurable.

La Fumec empezó a integrar a la industria, la academia y el gobierno en diversos temas, con los mems y NeMs como una de las áreas principales. En el informe bianual de la Fumec de 2002-2003 se fijó la siguiente estrategia global:

La Fundación ha centrado sus esfuerzos durante 2002 y 2003 para facilitar el conocimiento y la colaboración para el desarrollo de las acciones clave que pueden facilitar el desarrollo de clusters bi-nacionales de base tecnológica, en particular en la región Paso del Norte (Manufactura Avanzada, mems-Sistemas Micro Electromecánicos). Fumec ha apoyado los esfuerzos de los Laboratorios Nacionales Sandia, el Conacyt y los estados fronterizos, especialmente en la región Paso del Norte, para crear el Laboratorio Bi-Nacional de Sustentabilidad. 
Estos esfuerzos han expandido su impacto en otras regiones, como en el caso de las estrategias para desarrollar las capacidades de mems en México (Fumec, 2003: 40).

Los cambios en la orientación política de Fumec, dos años después de la muerte de su iniciador, el congresista estadounidense Brown en 1999, y en la aplicación de los fondos que pasaron de temas de sustentabilidad ambiental a la innovación tecnológica fueron determinantes en el desarrollo de la cadena de mems en México. El cuadro 1 resume las principales etapas y características.

\section{CUADRO 1}

Evolución de la orientación y características de Fumec

\begin{tabular}{cll}
\hline \multicolumn{1}{c|}{ Año } & \multicolumn{1}{c}{ Enfoque } & \multicolumn{1}{c}{ Características } \\
\hline 1993 & Creación & $\begin{array}{l}\text { Apoyo a agendas de I+D independien- } \\
\text { tes en los países receptores de tecnolo- } \\
\text { gía (filosofía política de E. Brown Jr.) }\end{array}$ \\
1993-2001 & $\begin{array}{l}\text { Proyectos en sectores de: salud pública, } \\
\text { sustentabilidad ambiental, problemas } \\
\text { socioeconómicos fronterizos y forma- } \\
\text { ción en cyt } \\
\text { Innovación tecnológica. mems priori- } \\
\text { tario }\end{array}$ & $\begin{array}{l}\text { Convenio con los Laboratorios Milita- } \\
\text { res Sandia }\end{array}$ \\
\hline
\end{tabular}

Fuente: Elaboración propia.

La integración de los SNL en los planes de acción de la Fumec y el impulso a actividades vinculadas más estrechamente a los negocios orientados a la investigación y comercialización de productos se debió en gran parte al contexto político guiado por el presidente mexicano Vicente Fox (2000-2006) y los ataques al World Trade Center en Nueva York en septiembre de 2001, con las consiguientes consecuencias en materia de seguridad.

La presidencia de Fox en México mostró una clara inclinación hacia el desarrollo de mercados libres, el papel de la empresa privada en el 
desarrollo y una integración más estrecha con Estados Unidos. En materia de сут mexicana, se aprobó el Programa Nacional de Ciencia y Tecnología 2001-2006, donde fue explícito el papel estratégico de la innovación y la сут para mejorar la competitividad internacional. Este programa, con la ley de сут de 2002, le dio mayor poder al Conacyt, al liberarlo de la supervisión de la Secretaría de Educación Pública (SEP), otorgarle un presupuesto independiente y garantizarle una serie de proyectos en diferentes sectores de la economía orientados hacia la mejora de los lazos entre la empresa privada y la $\mathrm{I}+\mathrm{D}$ financiada con recursos públicos (Lewis, 2006). Aunque la financiación de la investigación siempre ha sido escasa, el sector privado logró obtener $10 \%$ de los fondos de investigación del Conacyt en 2002, aumentando a 21\% en cuatro años (Sánchez, Martínez y Campos, 2009).

Con respecto a las relaciones internacionales, corporaciones y gobiernos de Estados Unidos, México y Canadá llevaron a cabo un intenso cabildeo en el primer lustro del siglo xxi hacia la profundización de la integración económica en el TLCAN vinculada con las preocupaciones de seguridad especificadas por el gobierno de Estados Unidos. Como resultado, a principios de 2005 la Asociación para la Seguridad y la Prosperidad de América del Norte fue firmada por los tres países. Es en este contexto que Fumec realiza acuerdos con los SNL con el objetivo de crear una agencia de negocios binacional llamada Laboratorio Binacional de Sustentabilidad.

El éxito en el BNSL y en otras iniciativas, así como la estrecha interacción con organizaciones gubernamentales e industriales clave en los Estado Unidos y México, dio credibilidad a la Fumec para trabajar con el Consejo de Competitividad de EU, y la Asociación de la Iniciativa para la Prosperidad, proporcionándonos la oportunidad de participar en el proceso de establecer una nueva visión binacional del papel de la innovación en el trabajo del Instituto Mexicano para la Competitividad. Durante este periodo el presidente Bush lanzó la Asociación para la Seguridad y la Prosperidad (Security and Prosperity Partnership, SpP) iniciativa que también incluye a Canadá; lo que es un paso crucial 
para avanzar en el comercio en una verdadera vía trinacional dentro de un marco que pone de relieve la seguridad (Fumec, 2005: 6).

Mientras que la ubicación del Bnsl en Nuevo México a unos pocos kilómetros de la frontera entre El Paso y Ciudad Juárez dio un peso importante a la función de los SNL por su proximidad a Albuquerque, éstos no eran la única parte interesada en el programa de MEMs impulsado por este laboratorio. En México, el presidente Fox fue explícito en su deseo de vincular el desarrollo de mems a la industria maquiladora que se estableció en México en el área de tecnologías de la información y la comunicación; éste fue un sector productivo que también tenía representación corporativa de Estados Unidos en el consejo de administración de la Fumec.

\section{PROGRAMA DE MICROSISTEMAS DE LA FUMEC}

Este programa propone estrategias para generar capacidades científico-tecnológicas y empresariales en grupos mexicanos para resolver problemas y necesidades de la industria. Estas estrategias se han enfocado en sectores industriales que tienen oportunidad para aplicaciones basadas en MEMS: automotriz, médico, energético y alimentario. Algunos resultados del programa son los siguientes (смM, s/f):

- Formación de recursos humanos especializados en MEMs, con un promedio de 120 investigadores, profesores, ingenieros con conocimientos en el tema, ubicados en 17 universidades y centros de investigación, en 7 estados de la Republica Mexicana. Esto se logró a partir de diferentes talleres y diplomados en tecnologías MEMS, algunos con sede en el INAOE.

- Creación de 10 Centros de Diseño de mems (2002) orientados al desarrollo de proyectos multidisciplinarios de innovación tecnológica basados en la aplicación de mems. 
- Creación del Laboratorio Binacional de Sustentabilidad (BNSL), una institución sin fines de lucro que propone negocios tecnológicos a lo largo de la frontera (2005).

- Colaboración para el establecimiento de la primera empresa de mems en México: team Technologies en 2008 (Fumec, 2008).

- Creación del Clúster Binacional de Encapsulado de mems en la Región Paso del Norte (Fumec, 2008).

- Creación de la Red de Laboratorios de Innovación de mems.

- Creación del Consorcio Mexicano de Microsistemas (смм) en 2007, como un spin-off de Fumec, tomando como referencia al Canadian Microsystem Corporation, para promover la competitividad de empresas y centros de investigación mediante el uso de los microsistemas (Fumec, 2010).

- Establecimiento de alianzas estratégicas con asociaciones internacionales de mems, como Micro, Nano, and Emerging Technologies Comercialization and Education Foundation (MANCEF) de Estados Unidos y el Canadian Microsystems Corporation (CMC), así como diversas Universidades de Estados Unidos y España.

Para enfrentar los retos antes propuestos, Fumec comenzó con la instalación de infraestructura para el desarrollo de los MEms en su primera etapa: el diseño. Para el diseño y simulación de éstos dispositivos se necesitan principalmente equipos de cómputo y software especializado CAD. A pesar de que se trata de la etapa de producción más barata, representa inversiones importantes de capital para mantener las licencias de uso del software.

En 2002, la Fumec, mediante un convenio con la Secretaría de Economía de México, lanzó una convocatoria para crear una red nacional de centros de diseño de mems llamada Red de CD-MEms. Esta iniciativa contemplaba, en sus inicios, solamente la región fronteriza; sin embargo, se decidió expandir la convocatoria a todo el país. Los centros de investigación seleccionados en la convocatoria se reunieron a finales de 2002 .

A inicios de 2003 se realizó una serie de reuniones con especialistas en mems de Estados Unidos, principalmente de los Laboratorios Sandia, 
ut-Arlington, Universidad de Nuevo México, ut-El Paso y algunas empresas estadounidenses especializadas en el diseño de estos sistemas, como memscap Inc. y Conventor. Todo esto con la finalidad de formar a los investigadores mexicanos en el campo de los mems (Fumec, 2003; Robles-Belmont, 2010). Cabe mencionar que no todos los centros de investigación seleccionados continuaron con el proyecto; sólo 10 formaron la Red de CD-MEMS: Centro de Investigación y Estudios Avanzados del Instituto Politécnico Nacional (Cinvestav), Guadalajara, Jalisco; Instituto de Investigaciones Eléctricas (IIE), Cuernavaca, Morelos; Instituto Nacional de Astrofísica Óptica y Electrónica (inaoe), Tonanzintla, Puebla; Universidad Autónoma de Ciudad Juárez (UACJ), Ciudad Juárez, Chihuahua; Universidad Veracruzana (uv), Boca del Río, Veracruz; Universidad Popular Autónoma del Estado de Puebla (upaep), Puebla, Puebla; Universidad Nacional Autónoma de México (UnAM), Distrito Federal; Instituto Tecnológico Superior de Irapuato (ITESI), Irapuato, Guanajuato; Instituto Tecnológico de Estudios Superiores de Monterrey (ITESm), Monterrey, Nuevo León; y Universidad de Guadalajara (udeg), Guadalajara, Jalisco.

También en 2003 se dio un acercamiento con el entonces presidente de México, Vicente Fox, en un taller organizado por la Fumec, el Conacyt y la transnacional Advanced Micro Devides (AMD) para promover la colaboración entre empresas hispánicas de base tecnológica en Estados Unidos y empresas, universidades y centros de investigación mexicanos, dentro del espíritu de colaboración con talentos mexicanos en el extranjero. En este encuentro, la FuMEC presentó la iniciativa del programa de desarrollo de mемs, en el cual el presidente de México mostró un especial interés. En una segunda reunión se promovió la iniciativa del BNSL, colaborando con los Laboratorios Sandia de Estados Unidos, orientado a fortalecer y explotar el potencial de la región fronteriza para generar clústeres binacionales de innovación; por ejemplo, la región Paso del Norte que comprende Ciudad Juárez, Chihuahua, El Paso, Las Cruces y Santa Teresa (Robles-Belmont, 2010; Fumec, 2004).

En 2004 se crea el Centro de Articulación Productiva de mems (CAP-MEMS). Este centro genera actividades en México para impulsar e 
implementar la Estrategia de Tecnologías mems. Nació como un derivado de la Fumec con el objetivo de facilitar los vínculos entre empresarios, académicos y tomadores de decisiones con el fin de generar colaboraciones para el desarrollo de nuevos productos y negocios (Fumec, 2003).

Los acercamientos de la Fumec con el presidente de México dan frutos en el 2005, primeramente con la creación de la red de Laboratorios de Innovación en MEMS (LI-MEMS) y después con el BNSL. La primera, creada a fines de 2005, estuvo integrada originalmente por tres instituciones; después se incluiría una cuarta, con el fin de cubrir la cadena de valor de mems centrados en la prueba y caracterización de éstas, desarrollo de prototipos, encapsulado y validación (Fumec, 2005). La segunda, con sede en Alburquerque, Nuevo México, tuvo como misión construir negocios fronterizos basados en tecnología para el desarrollo económico de la región. Este laboratorio tiene la característica importante de ser financiado por ambos gobiernos, aunque opera en Estados Unidos. La principal acción de este centro es la creación del Clúster de Empaquetado del Paso del Norte, formado por instituciones académicas, laboratorios de investigación gubernamentales y empresas, con la finalidad de investigación, desarrollo de productos y comercialización para el empaquetado de MEMS, principalmente para aplicaciones en sistemas automotrices, biomédicos y telecomunicaciones. Los integrantes de este clúster son UT-El Paso, New Mexico State University, New Mexico Institute of Technology, El Paso Community College, Tvi College en Albuquerque, Laboratorios Nacionales Sandia, Laboratorios de defensa White Sands Missile Range, Delphi y el вnsL en Estados Unidos; por el lado de México, el Centro de Investigación de Materiales Avanzados (Cimav), la Universidad Autónoma de Ciudad Juárez y el Instituto Tecnológico de Estudios Superiores de Monterrey-Campus Juárez (Maxwell, 2006).

El proyecto del clúster de Empaquetamiento de Paso del Norte es un corredor industrial que va desde Chihuahua, México, hasta Alburquerque, Nuevo México, en Estados Unidos. Una de las compañías beneficiadas con este proyecto es Team Technologies, ubicada en Nuevo México, con instalaciones para fabricación de mems en Ciudad Juárez, 
Chihuahua, convirtiéndose en la primera compañía en México con esta capacidad.

En abril de 2007, el CAP-MEMs se consolida y se da paso al Consorcio Mexicano de Microsistemas (СмM), el cual siguió siendo un derivado de FUMEC basado en un modelo canadiense de operación. El trabajo del Смм es la identificación de oportunidades en el área de los microsistemas a fin de implementar una solución basada en MEMs en nichos de oportunidad.

En la actualidad, el смм — con apoyo de la Fumec- se encarga de cuatro redes del programa de MEMs (Fumec, 2008):

- La Red de especialistas en MEMs, conformada por 20 instituciones educativas y 260 profesores-investigadores. Esta red, comenzó desde los inicios del programa de MEMs de FUMEC. Se fue consolidando con los años a través de diplomados de especialización, conferencias, redes de colaboración y proyectos conjuntos entre investigadores de diferentes instituciones apoyadas por Conacyt y los Consejos de Ciencia y Tecnología estatales con el fin de promover el intercambio y la capacitación académica, es decir, cerrar la brecha de falta de recursos humanos especializados en MEMs.

- La Red de CD-MEMs, de la cual 10 instituciones forman parte. Esta red cubre la necesidad de la primera etapa de la cadena de valor de MEMS: el diseño y modelado. En la figura 1 se muestra un mapa de la distribución por Estado de los centros de la Red CD-mems.

- La Red de LI-MEMS, actualmente compuesta por 4 instituciones. Con apoyo de la Secretaría de Economía de México está dedicada a atacar el segundo reto de la Fumec: la construcción de capacidades tecnológicas para el desarrollo de mems. En México sólo el sector gubernamental tiene los recursos para invertir en el tipo de infraestructura necesaria, ya que se requiere de cuartos limpios, los cuales, a veces, superan en costo los equipos que se tendrán dentro de ellos. Con el fin de brindar servicios que contribuyan en la cadena de valor de estos sistemas se desarrollaron los siguientes centros (смм, s/f):

a) Li-mems (Prototipos y Prueba). Este laboratorio tiene sede en Puebla, en el INAOE; ofrece servicios de prueba y fabricación de MEMS. 
FIGURA 1

Distribución por Estado de los centros de la Red de CD-MEMS

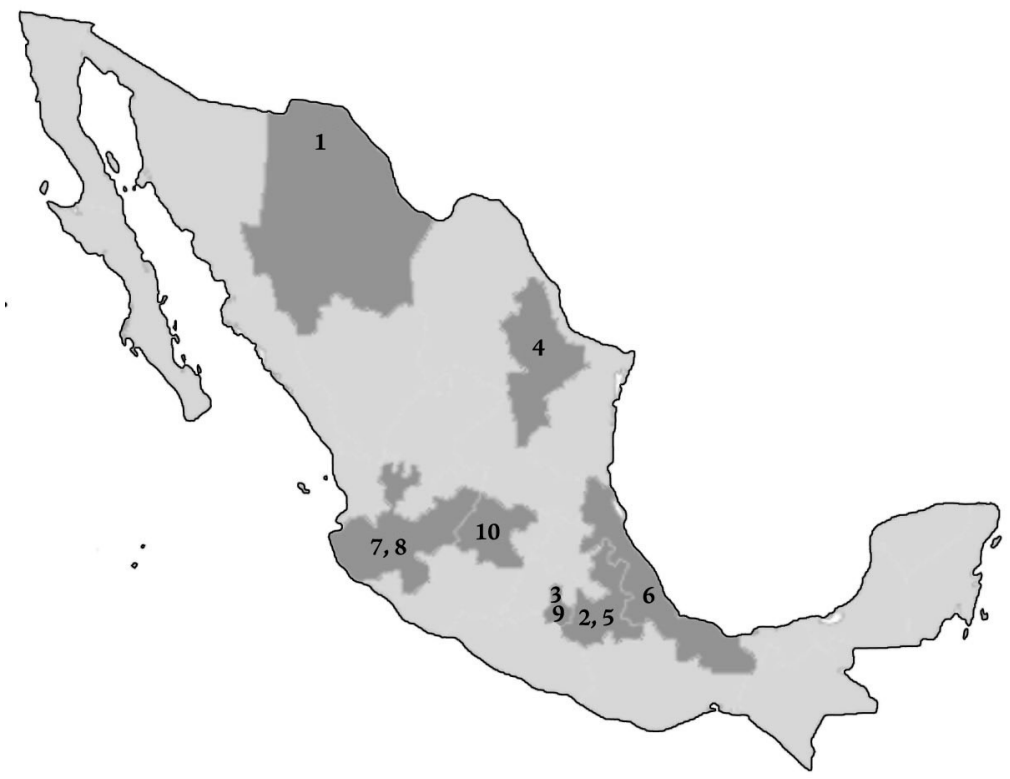

Red CD-MEMS

1. L’ACJ (Universidad Autónoma de Ciudad Juárez), Ciudad Juárez. Chihuahua.

2. InAoe (Instituto Nacional de Astrofísica Óptica y Electrónica). Tonanzintla. Puebla.

3. unAm (Universidad Nacional Autónoma de México). Distrito Federal.

4. itesm (Instituto Tecnológico de Estudios Superiores de Monterrey). Monterrey. Nuevo León.

5. upaep (Universidad Popular Autónoma del Estado de Puebla). Puebla. Puebla.

6. uv (Universidad Veracruzana). Boca del Río. Veracruz.

7. Cinvestav (Centro de Investigación y Estudios Avanzados del Instituto Politécnico Nacional). Guadalajara. Jalisco.

8. U de G (Universidad de Guadalajara). Guadalajara, Jalisco.

9. IIE (Instituto de Investigaciones Eléctricas). Cuernavaca. Morelos.

10. itesi (Instituto Tecnológico Superior de Irapuato). Irapuato. Uuanajuato.

Fuente: elaboración propia.

b) LI-MEMs (Encapsulado de MEMS). Se encuentra en el Centro de Investigación en Ciencia y Tecnología Aplicada (сістA), de la uACJ en Ciudad Juárez, Chihuahua.

c) LI-MEMS (Validación de MEMS). Ubicado en la upaep, cuenta con los servicios de validación y pruebas de confiabilidad de mems. 
Existen otros laboratorios destacados en vías de consolidación que Fumec ha impulsado para ofrecer servicios en el tema de mems: Centro de Investigación de Micro y Nanotecnología (microna), de la uv en Boca Del Río, Veracruz, y Centro de Nanociencia y Micro y Nanotecnología del Instituto Politécnico Nacional, unidad Zacatenco, en el Distrito Federal.

- La Alianza Estratégica y Red de Innovación para la Competitividad (AERI). Una red de colaboración fortalecida por el Conacyt, la Fumec y el смм con el fin de crear sinergias entre empresas, universidades e instituciones de investigación para realizar proyectos innovadores y competitivos en las empresas con del objetivo de promover el desarrollo económico (Fumec, 2010).

\section{CONCLUSIONES}

Cada vez más, los mems están relacionados con las nanotecnologías; en consecuencia, en Estados Unidos el financiamiento federal en la materia incluye a la investigación. Los MEMs requieren una infraestructura y equipos similares a los de las nanotecnologías, y hay una tendencia natural a la miniaturización que convertirá a los MEMS en NEMS en el largo plazo. Los MEMS son ampliamente utilizados en la industria del automóvil y la industria de los dispositivos de comunicación y recreación, además de muchos otros sectores económicos. Su mercado mundial es de alrededor de 9 mil millones de dólares.

La cadena de valor de мемs permite que las primeras etapas se hagan virtualmente, o como prototipos, mucho antes de que lleguen a la producción en serie. Por los altos costos involucrados en la producción de MEMs, la producción en masa requiere de instalaciones especiales y costosas, que hoy sólo las tienen las grandes empresas transnacionales. Esto sugiere que probablemente los recién llegados al campo de estos sistemas se especialicen en las primeras etapas de actividades de bajo costo; actividades que se subordinan a las necesidades de las grandes corporaciones que convierten pruebas virtuales o prototipos en productos finales. 
Un camino paralelo y distinto se encuentra en la investigación de MEMS para fines militares, ya que lo militar puede no requerir una producción a gran escala, ni tener límites de costo y rentabilidad. Los MEMS son una tecnología de doble uso, que permite un desarrollo simultáneo en áreas civiles y militares. El hecho de que la iniciativa de mems en México fue asociada, desde el principio, con los Laboratorios Sandia de orientación militar de Estados Unidos plantea la interrogante de si los intereses militares estadounidenses incidirán en la agenda de investigación de mems en México.

El desarrollo de mems en México, fomentado por Fumec, refleja las dos rutas: el camino hacia la industria civil de información y automotriz, ambas dominadas en México por las grandes corporaciones transnacionales, y la vía fincada en la asociación entre los snl y Conacyt. Al mismo tiempo y en menos de una década, México ha desarrollado un importante capital humano en tecnología MEMS, así como redes tecnológicas en México, en Estados Unidos y con otros socios extranjeros. Asimismo, México también ha desarrollado una importante infraestructura para el desarrollo de los мемs; un logro impresionante, dado el hecho de que hace poco más de una década prácticamente no había nada de tecnología mems en el país. Sin embargo, el fuerte lazo México-Estados Unidos que se ha desarrollado para impulsar a esta tecnología en México implica, aparentemente, una subordinación a las necesidades de las corporaciones transnacionales o las necesidades militares de Estados Unidos. Esto llama a hacer un examen más detenido de cómo esta importante tecnología se desarrollará en los términos de las propias aspiraciones y crecimiento económico de México.

Es muy pronto para prever si la infraestructura y capacidad de México en los MEMS se integrará a la industria del automóvil y los semiconductores, como Fumec lo visualiza para los primeros años de este siglo. No obstante, se demuestra que, con voluntad política, México puede mejorar significativamente sus condiciones de investigación científica. 


\section{BIBLIOGRAFÍA}

Anónimo (2007), «What is mems Technology?», 2ic.cn, disponible en: http:// www.2ic.cn/thread-327920-1-1.html (Fecha de consulta: 15 abril de 2013).

Banks, Danny (2006), Microengineering, MEMs, and Interfacing: A Practical Guide, London, New York, CrC Press, disponible en: http://robochamp.ru/files/ PDF/Microengineering\%20MEMs\%20and\%20Interfacing\%20-\%20Danny\%20Banks.pdf.

Brown, George (1993), «The mother of necessity: technology policy and social equity», en Remarks of Congressman George E. Brown Jr. Chairman, Washington DC, Committee on Science, Space, and Technology. AAAs Science and Technology Policy Colloquium.

\& Daniel Sarewitz (1991), «Fiscal alchemy: Transforming debt into research», Issues in Science and Technology, vol. 8, núm. 1.

Consorcio Mexicano de Microsistemas (s/f), «Tecnología mems (Sistemas Micro Electro Mecánicos)», Consorcio mexicano de microsistemas, disponible en: http://www.cmm.org.mx/ (Fecha de consulta: 16 de abril de 2013).

Da Silva, M. G., R. Giasolli, S. Cunningham y D. DeRoo (2002), «mems Design for Manufacturability (DFM)», Sensors Expo E Conference 2002, disponible en: http://wenku.baidu.com/view/8e671278168884868762d685.html (Fecha de consulta: 16 de abril de 2013).

Department of Defense (1995), «Microelectromechanical Systems Opportunities», Department of Defense, Dual-use Technology Industrial Assessment, disponible en: http://www.dtic.mil/cgi-bin/GetTRDoc?AD=ADA304675 \&Location $=\mathrm{U} 2 \&$ doc $=$ GetTRDoc, pdf (Fecha de consulta: 16 de abril de 2013).

Fedder, Gary (1999), «Structured Design Methodology for Mems», Carnegie Mellon University, disponible en: http://www.design.caltech.edu/NSF_ MEMS_Workshop/fedder.pdf (Fecha de consulta: 16 de abril de 2013).

Freiburghouse, Andrew (2001), «The Mems MIcrocosm: Military», Forbes, disponible en: http://www.forbes.com/asap/2001/0402/052.html (Fecha de consulta: 16 de abril de 2013).

Fumec (1997), Reporte de actividades 1993-1997, Fundación México-Estados Unidos para la Ciencia, disponible en: http://fumec.org.mx/v6/htdocs/informe1993_1997.pdf (Fecha de consulta: 16 de abril de 2013). 
(1999), Reporte de actividades 1998-1999, Fundación México-Estados Unidos para la Ciencia, disponible en: http://fumec.org.mx/v6/htdocs/informe1998_1999.pdf (Fecha de consulta: 16 de abril de 2013).

(2002), Reporte de actividades 2000-2001, Fundación México-Estados Unidos para la Ciencia, disponible en: http://fumec.org.mx/v6/htdocs/informe2000_2001.pdf (Fecha de consulta: 16 de abril de 2013).

(2003), Reporte bianual de actividades 2002-2003, Fundación MéxicoEstados Unidos para la Ciencia, disponible en: http://fumec.org.mx/v6/ htdocs/informe2002_2003.pdf (Fecha de consulta: 16 de abril de 2013).

(2005), Reporte bienal de actividades 2004-2005, Fundación México-Estados Unidos para la Ciencia, disponible en: http://fumec.org.mx/v6/htdocs/ informe2004_2005.pdf (Fecha de consulta: 16 de abril de 2013).

(2008), Reporte bienal de actividades 2006-2007, Fundación México-Estados Unidos para la Ciencia, disponible en: http://fumec.org.mx/v6/htdocs/ informe2006_2007.pdf (Fecha de consulta: 16 de abril de 2013).

(2010), Reporte bienal de actividades 2008-2009, Fundación México-Estados Unidos para la Ciencia, disponible en: http://fumec.org.mx/v6/htdocs/ RepEsp_0809.pdf (Fecha de consulta: 16 de abril de 2013).

Gandhi, Prasanna (s/f), «Suman Mashruwala. Advanced $\mu$ icroengineering Laboratory», disponible en: http://www.me.iitb.ac.in/ mems/sml/sml.pdf (Fecha de consulta: 16 de abril de 2013).

Lawton, Russel et al. (1999), mems Reliability for Critical and Space Applications, Society of Photo Optical.

Lewis, James (2006), National Policies for Innovation and Growth in Mexico, Washington, Center for Strategic and International Studies.

Maluf, Nadim (2000), An Introduction to Microelectromechanical Systems Engineering, Boston, Artech House.

Materials Modification Inc ( $/ \mathrm{f})$, «Materials Modification Inc», disponible en: http://www.matmod.com/FaQ.html (Fecha de consulta: 16 de abril de 2013).

Matsumoto, Craig (1999), «Sandia pushes for mems commercialization», EETimes News E Analysis, en: http://eetimes.com/electronics-news/4168626/ Sandia-pushes-for-MEMs-commercialization (Fecha de consulta: 16 de abril de 2013).

Maxwell, Paul (2006), «Bnsl The Bi National Sustainability Laboratory», ponencia presentada en NARC Conference, Corpus Christi, Tx, disponible en: 
http://narc,org/uploads/File/Transportation/Freight\%20Summit/ Maxwell_BnsL.pdf (Fecha de consulta: 16 de abril de 2013).

McBrayer, J. D. (2000), «The transfer of disruptive technologies: lessons learned from Sandia National Laboratories», IEeEXplore, Presented at the Proceedings of the 2000 IEEE, Engineering Management Society, disponible en: http: / ieeexplore.ieee.org/xpl/login.jsp?tp $=$ Karnumber $=872525 \&$ url $=$ http $\% 3 \mathrm{~A} \% 2 \mathrm{~F} \% 2$ Fieeexplore.ieee.org\%2Fxpls\%2Fabs_all.jsp\%3 Farnumber\%3D872525 (Fecha de consulta: 16 de abril de 2013).

MNX (s/f), «MEMs and Nanotechnology Applications. mEMs E Nanotechnology Exchange», disponible en: https://www.mems-exchange.org/mems/applications.html (Fecha de consulta: 16 de abril de 2013).

Muknerjee, Tamal (2003), «Mems design and verification», Teste Conference, Proceedings. ITC 2003. International.

Nano.gov (s/f), «What's So Special about the Nanoscale?», disponible en: http:// www.nano.gov/nanotech-101/special (Fecha de consulta: 16 de abril de 2013). ¿Cómo debe quedar esto?

Plunkett (2010), Plunkett's Nanotechnology E MEMs Industry Almanac, Plunkett Research.

Prime Faraday (2002), «An Introduction to MEms (Micro-electromechanical Systems)», Prime Faraday Technology Watch, disponible en: http://www.lboro. ac.uk/microsites/mechman/research/ipm-ktn/pdf/Technology_review/ an-introduction-to-mems.pdf (Fecha de consulta: 16 de abril de 2013).

RheA, John (2000), «MEMS: following in the footsteps of the Internet?», Military E Aerospace Electronics, disponible en: http://www.militaryaerospace.com/ articles/print/volume-11/issue-9/departments/report-from-washingtonand-elsewhere/mems-following-in-the-footsteps-of-the-internet.html (Fecha de consulta: 16 de abril de 2013).

Robles-Belmont, Eduardo (2010), «Las Fundaciones en el Desarrollo de Tecnologías Emergentes: Desarrollo de los mems en México», Jornadas Latinoamericanas de Estudios Sociales de la Ciencia y Tecnología, Buenos Aires, disponible en: http://www.esocite2010.escyt.org/sesion_ampliada.php?id_ Sesion $=63$ (Fecha de consulta: 16 de abril de 2013).

SÁnchez, Germán, Ma. Eugenia Martínez y Guillermo Campos (2009), «iMéxico en la economía y sociedad del conocimiento? Una revisión a las políticas públicas», en Germán Sánchez (ed.), América Latina y el Caribe en la economía 
y sociedad del conocimiento, México, Consejo Latinoamericano de Ciencias Sociales /Benemérita Universidad Autónoma de Puebla, disponible en: http://www.ceipil.org.ar/wp-content/uploads/2009/12/libro.pdf (Fecha de consulta: 16 de abril de 2013).

Sandia National Laboratories (s/f), «Sandia National Laboratories: About Sandia», disponible en: http://www.sandia.gov/about/ (Fecha de consulta: $16 \mathrm{de}$ abril de 2013).

(2001a), «Sandia, Ardesta join forces to commercialize mems and Microsystems», News Releases, disponible en: http://www.sandia.gov/media/ NewsRel/NR2001/ardesta.htm (Fecha de consulta: 16 de abril de 2013). (2001b), SUMMiT V Overview [¿Esto qué es?]. Sandia National Laboratories, disponible en: http $\$ / /$ mems.sandia.gov/tech-info/summit-v.html (Fecha de consulta: 16 de abril de 2013).

(13 de diciembre de 2005), «BiNational Sustainability Laboratory opens, hopes to create 'necklace of labs' along Mexican border». EurekAlert!, disponible en: http://www.eurekalert.org/pub_releases/2005-12/dnl-bs1121205. php (Fecha de consulta: 16 de abril de 2013).

Senturia, Stephen (2000), Microsystem Design, Springer.

Wilson, J. R. (2003), «Smart munitions development relies heavily on MEms technology», Military $\mathcal{E}$ Aerospace Electronics, disponible en: http://www. militaryaerospace.com/articles/print/volume-14/issue-1/features/specialreport/smart-munitions-development-relies-heavily-on-mems-technology. html (Fecha de consulta: 16 de abril de 2013).

Yolé (2011), «Top 30 mems Companies 2010: Big four capture a third of the total MEMS market», disponible en: http://www.yole.fr/iso_upload/ News/2011/Top30memsCompanies2010_April2011.pdf (Fecha de consulta: 16 de abril de 2013 ). 
